\title{
Balancing Efficiency and the Protection of Research Participants: Canadian Allergy/Asthma Researchers' Perspectives on the Ethics Review of Multi-Site Health Research
}

\author{
Zubin Master ${ }^{1}$, Nola M. Ries ${ }^{2}$ and Timothy Caulfield ${ }^{2 *}$
}

${ }^{1}$ Health Law and Science Policy Group, Rm 462, Law Centre, University of Alberta, 89 Avenue and 111 Street, Edmonton, AB, T6G 2H5, Canada ${ }^{2}$ LLM, Health Law and Science Policy Group, Law Centre, University of Alberta, 89 Avenue and 111 Street, Edmonton, AB, T6G 2H5, Canada

\begin{abstract}
Many studies examining several aspects of multi-institutional research ethics review and the opinions of different stakeholders show the current process to be resource intensive and inefficient. We discuss the experiences and opinions of individual Canadian allergy/asthma researchers engaged in multi-centre research and find that most researchers are dissatisfied, perceive the process as ineffective, and strongly desire some type of reform. These results highlight the tension between efficiency in research ethics review and the effective protection of subjects. Harmonization initiatives and alternate review strategies are being implemented throughout Canada and should help increase efficiency while not compromising the protection of research subjects. With time, evidence gathered from empirical studies that evaluate these new strategies will help determine the effectiveness of the various reform strategies for multi-site ethics review.
\end{abstract}

Keywords: Research ethics review; Multi-site health research; Allergy/asthma researchers; Researcher perspectives/views; Research Ethics Boards (REBs); Variability in multi-site ethics review; Harmonization/standardization

Abbreviations: REB: Research Ethics Board; TCPS: Tri-Council Policy Statement: Ethical Conduct for Research Involving Humans

\section{Introduction}

A number of issues have been identified in the ethics review of research performed at multiple sites including the possibility of introducing unnecessary inefficiencies to the research process [1], escalating the costs of carrying out research [2], deterring participation in a national research network [3], increasing the complexity of consent forms $[2,4,5]$, and delaying study timelines [2,6,7], while not necessarily improving protocols [4]. This could be due to local research ethics boards (REBs) disagreeing on various aspects of research ethics and differences among REB members in interpreting guidelines and policies $[8,9]$. As in other jurisdictions, the potential challenges with ethics review of multi-site studies have been a matter of concern in Canada [10]. Although the perception of many researchers and others is that the research ethics review process for multi-site research is inefficient, it must be remembered that the core mandate of REBs is the protection of human subjects, not the facilitation of research [11].

To understand better some of the challenges researchers might face in Canada, we conducted a survey of researchers in the Canadian Allergy, Genes and Environment Network of Centres of Excellence (AllerGen) (www.allergen-nce.ca). A key aspect of AllerGen's activities is the conduct of multi-centre studies across the country. One example is a large birth cohort study - the Canadian Healthy Infant Longitudinal Development Study - with study sites in four provinces. The survey asked about researcher experiences with multi-site applications and researcher opinions about research ethics reform. Though the sample was small $(n=30)$ and response rate modest $(16 \%)$, the results contribute to the literature on researchers' experiences. While researcher perspectives are just one of many considerations to take into account in the reform of REB processes and policies, understanding researcher opinions can help ensure that reform initiatives produce the most effective system of research ethics review that protects subjects while reducing unnecessary inefficiencies.

\section{The Survey Results}

What did we find?Manyrespondents (69\%) were somewhat dissatisfied or very dissatisfied with their experience submitting multisite ethics applications with only one participant being very satisfied (Figure 1). All respondents experienced variation among REBs including the need to fill out different forms and delays in response from REBs. Specifically, REB variation was highest in informed consent requirements, followed closely by participant recruitment and slightly less variation was seen in requirements for maintaining confidentiality and the interpretation of risk (Figure 1). To address differing REB requirements, most research investigators in our study modified the protocol either at one or all study sites (Figure 1). Respondents also reported that the multi-site review process resulted in delays in recruiting subjects, involving trainees (e.g., students, post-doctoral fellows, residents), and hiring research or other staff (Figure 2).

The opinions of AllerGen researchers are consistent with perspectives of other Canadian and international researchers engaged in multi-site research. Other studies have reported variability in regards to consent $[4,12,13]$, the assessment of risk [8,9,14], and the time it takes to review protocols $[15,16]$. Eric Racine's group has recently documented neuroimaging researchers' perspectives outlining inconsistencies in the input between multiple Canadian REBs, delays in protocol approval, and has also shown variability in REB approved consent forms for neuroimaging research [17-19].

${ }^{*}$ Corresponding author: Timothy Caulfield, LLM, FRSC, Health Law and Science Policy Group, Rm 461, Law Centre, University of Alberta, 89 Avenue and 111 Street, Edmonton, AB, T6G 2H5, Canada, Tel: 780-492-8358; Fax: 780-492 9575; E-mail: tcaulfld@law.ualberta.ca

Received August 23, 2011; Accepted September 20, 2011; Published September 25, 2011

Citation: Master Z, Ries NM, Caulfield T (2011) Balancing Efficiency and the Protection of Research Participants: Canadian Allergy/Asthma Researchers Perspectives on the Ethics Review of Multi-Site Health Research. J Clinic Res Bioeth 2:104e. doi:10.4172/2155-9627.1000104e

Copyright: ( 2011 Master Z, et al. This is an open-access article distributed unde the terms of the Creative Commons Attribution License, which permits unrestricted use, distribution, and reproduction in any medium, provided the original author and source are credited. 
Citation: Master Z, Ries NM, Caulfield T (2011) Balancing Efficiency and the Protection of Research Participants: Canadian Allergy/Asthma Researchers' Perspectives on the Ethics Review of Multi-Site Health Research. J Clinic Res Bioeth 2:104e. doi:10.4172/2155-9627.1000104e

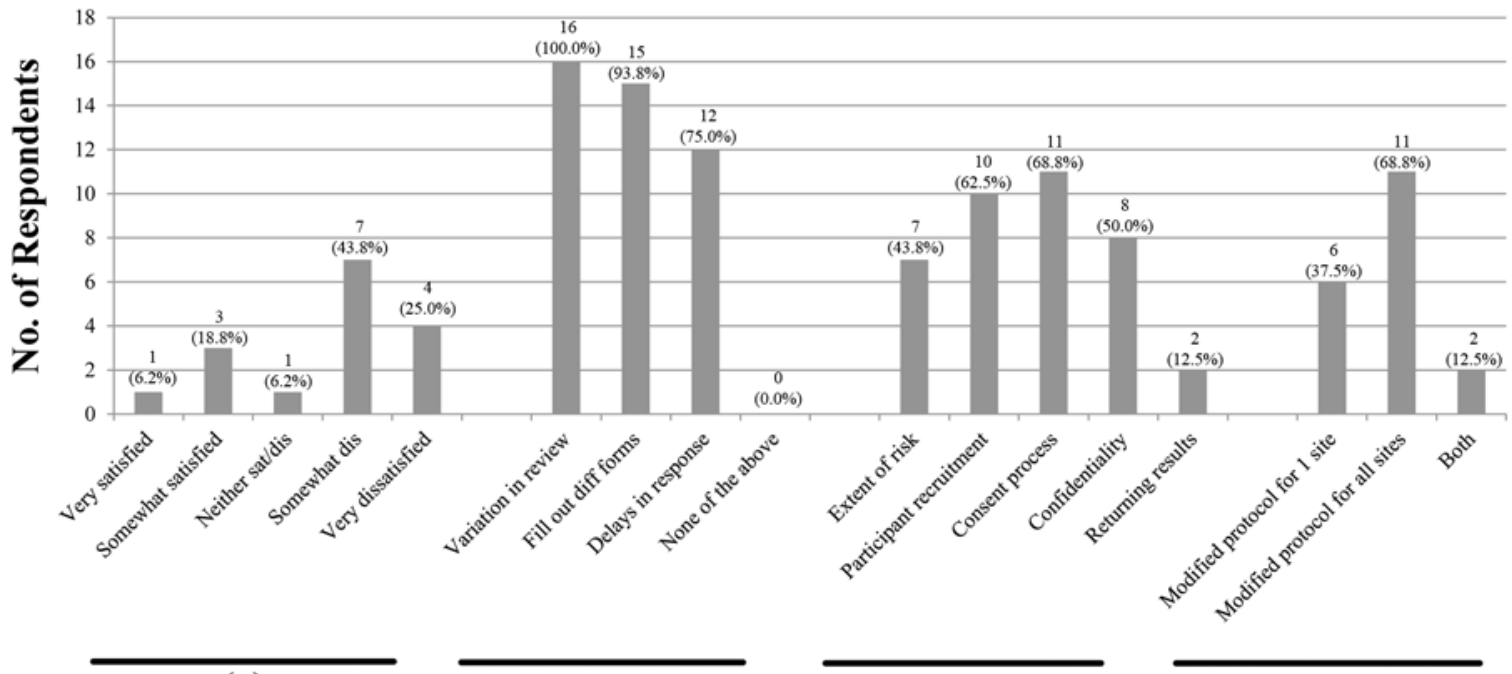

(a) (b) (c) (d)

\section{Survey Responses to Questions}

Figure 1: Researchers' Experience with Ethics Review of Multi-Site Studies. Responses of AllerGen researchers ( $n=16$ ) to four multiple choice questions regarding their experience with the submission of ethics applications to multiple REBs were quantified. Questions: (a) Overall, how would you describe your experiences with the REB ethics review process of multi-site research projects? (b) Have you encountered one or more of the following experiences with the REB ethics review process of multi-site research projects? (c) What aspects of the study received different responses from REBs? (d) How did you reconcile the different responses provided by different REBs for the same multi-site research study?

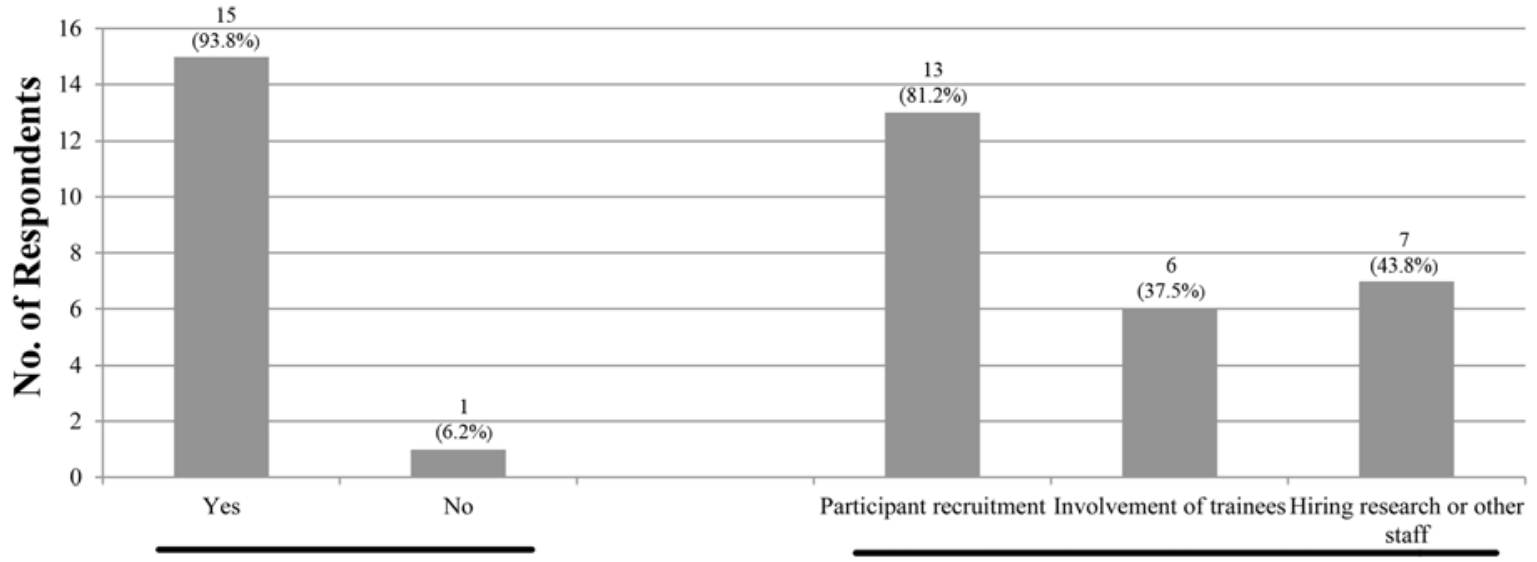

(e)

(f)

\section{Survey Responses to Questions}

Figure 2: Researchers' Experience with Delays in Research Due to REB Ethics Review of Multi-Site Studies. Responses of AllerGen researchers ( $=16$ ) to 2 multiple choice questions regarding their experience with the submission of ethics applications to multiple REBs was quantified. Questions: (e) Has your study start date ever been delayed by the requirement to seek ethics approval from multiple REBs? (f) Did the time required to obtain multiple REB approvals for a multi-site study delay any of the following?

\section{Researchers' Views on Reform Strategies}

Despite the stated concerns about multi-site review, to our surprise, over half of the researchers felt that it is very or somewhat important for individual REBs to conduct their own review while the remainder believed independent review was less important (Figure 3). This perception might be informed by the fact that the primary Canadian research ethics policy, the Tri-Council Policy Statement: Ethical Conduct for Research Involving Humans (TCPS),states that an institution "may approve alternative review models for research involving multiple REBs" but it "remains responsible for the ethical acceptability and ethical conduct of research undertaken within its jurisdiction or under its auspices irrespective of where the research is conducted" (Article 8.1) [20]. The practice of having every institution do an individual REB review has been a long tradition in Canada and legal liability concerns about accepting the review of another REB reinforce the practice of multiple reviews. The TCPS does, however, suggest alternative review models for multi-site studies, with an aim "to provide flexibility and efficiency, and avoid unnecessary duplication of review without compromising the protection of participants" (page 99) [20]. 
Citation: Master Z, Ries NM, Caulfield T (2011) Balancing Efficiency and the Protection of Research Participants: Canadian Allergy/Asthma Researchers' Perspectives on the Ethics Review of Multi-Site Health Research. J Clinic Res Bioeth 2:104e. doi:10.4172/2155-9627.1000104e

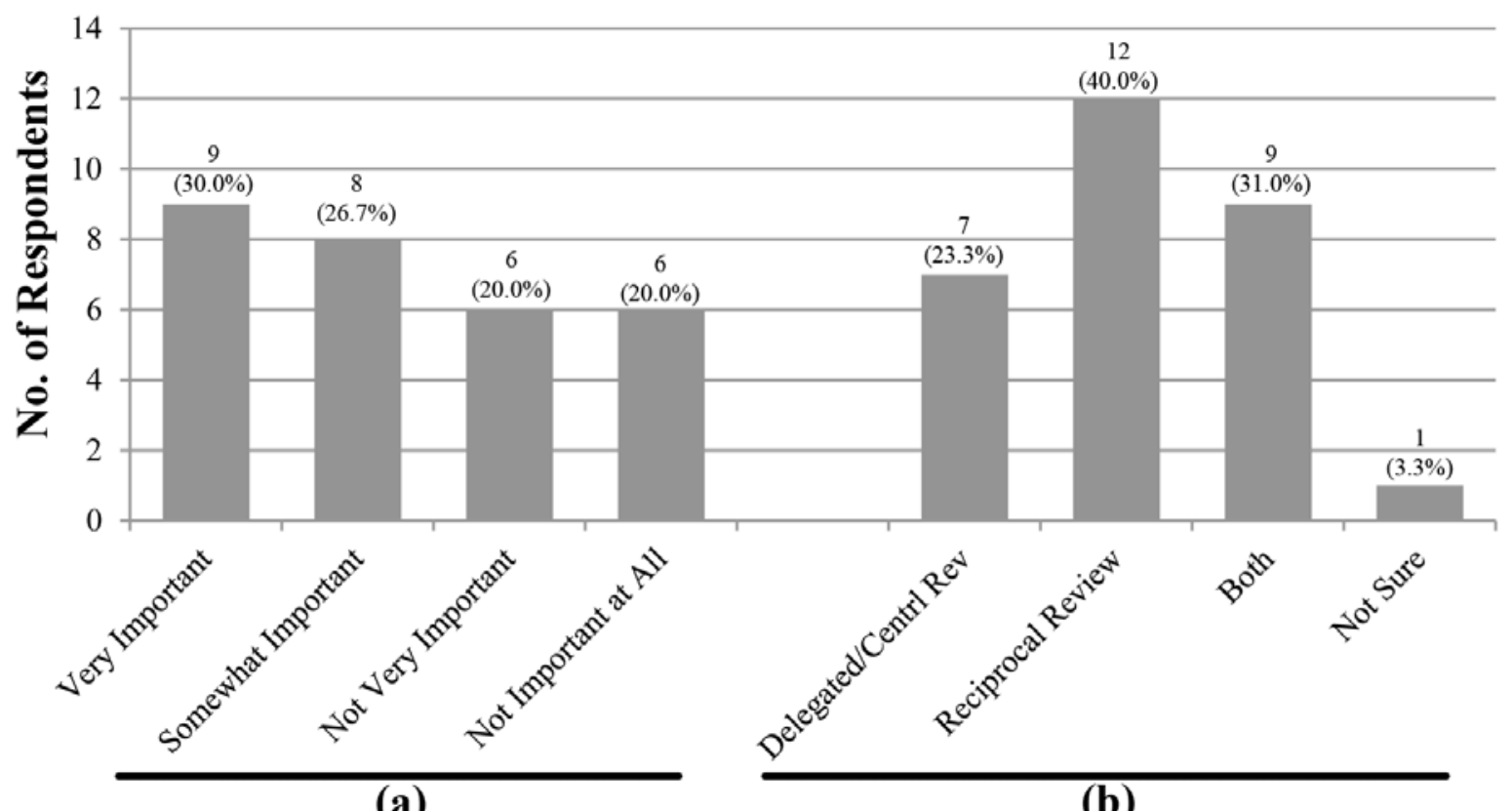

(a)

(b)

\section{Survey Responses to Questions}

Figure 3: Researchers' Views on Reform Strategies of Ethics Review of Multi-Site Studies. Responses of AllerGen researchers ( $n=30$ ) to 2 multiple choice questions regarding their experience with reform strategies of ethics review of multi-site studies was quantified. Questions: (a) How important is it for each REB to conduct its own review of a multi-site study protocol (instead of, for example, accepting the review of another REB)? (b) What type of reform strategy do you feel might streamline REB review for multi-site studies?

Our survey asked researchers about strategies for reforming multisite review. Almost all respondents (97\%) selected one or more reform strategy. Clearly, researchers think something needs to be done to streamline the process (Figure 3). Many researchers felt that either a reciprocal REB review process (i.e., where REBs agree on a process by which they will accept another's decision) or delegated review to a specialized, central, or multi-institutional REB, or both, would streamline REB review (Figure 3).

\section{Conclusions}

While the study is small, the results serve to provide insight into the perspectives of individual Canadian allergy/asthma researchers engaged in biomedical, drug related, and social science research and the findings align with other research on point. They highlight researchers' concerns with the multi-site research ethics review process. Researchers seem to feel that REBs are inappropriately inefficient and that multisite processes add complexity (e.g. multiple forms, variation in reviews) and delay research activity (e.g. recruiting subjects and hiring staff).

Of course, these concerns must be balanced against the primary function of ethics review: the protection of research participants. While multiple review processes may slow the research process in some cases, there could be benefits if one REB identifies and proposes solutions for an ethical issue that is not raised by other REBs that review the study [21]; redundancy could be an important element that protects participants and promotes integrity in the research ethics review process. It is, however, likely that research ethics review for multi-site studies can benefit from different strategies that could make the overall process more efficient.

Several strategies to reduce variability, ensure consistency, and perform high quality ethics review have been suggested in the literature and, in some cases, implemented in practice (reviewed in Caulfield et al) [10]. Harmonization initiatives, i.e., the development of standardized forms, having an open and on-going dialogue between researchers and REBs, or the development of a centralized or specialized REB, all serve as potential strategies to reduce variability, ensure the efficient use of resources and lessen administrative burdens on REBs, while maintaining appropriate protection for participants. These emerging strategies provide an opportunity to research both the opinions of stakeholders and the effectiveness and efficiency of various research ethics reform initiatives. Research of this kind seems the only way to craft a system that is not unnecessarily burdensome or time consuming and that keeps the welfare of the research participant as the paramount consideration.

\section{Acknowledgements}

The research was possible due to a generous grant from the Allergy, Genes and Environment Network of Centres of Excellence Inc. (AllerGen). We would like to acknowledge Christen Rachul for administering the survey, all of the participants who completed the survey, and the AllerGen conference organizers who graciously facilitated the distribution of our survey at the 2011 annual meeting. The authors would also like to thank Nina Hawkins for administrative support. The work presented here does not represent the views of Health Canada or the Canadian government.

\section{References}

1. Menikoff J (2010) The paradoxical problem with multiple-IRB review. N Engl J Med 363: 1591-1593.

2. Ravina B, Deuel L, Siderowf A, Dorsey ER (2010) Local IRB review of multicenter trial: Local Costs without Local Context. Ann Neurol 67: 258-260.

3. Finch SA, Barkin SL, Wasserman RC, Dhepyasuwan N, Slora EJ, et al. (2009) Effects of local institutional review board review on participation in nationa 
Citation: Master Z, Ries NM, Caulfield T (2011) Balancing Efficiency and the Protection of Research Participants: Canadian Allergy/Asthma Researchers' Perspectives on the Ethics Review of Multi-Site Health Research. J Clinic Res Bioeth 2:104e. doi:10.4172/2155-9627.1000104e

practice-based research network studies. Arch Pediatr Adolesc Med 163:11301134.

4. Burman W, Breese P, Weis S, Bock N, Bernardo J, et al. (2003) The effects of local review on informed consent documents from a multicenter clinical trials consortium. Control Clin Trials 24: 245-255.

5. Paasche-Orlow MK, Taylor HA, Brancati FL (2003) Readability standards for informed-consent forms as compared with actual readability. $\mathrm{N}$ Engl $\mathrm{J}$ Med 348: $721-726$.

6. Burman WJ, Reves RR, Cohn DL, Schooley RT (2001) Breaking the camel's back: multicenter clinical trials and local institutional review boards. Ann Intern Med 134: 152-157.

7. McNay LA, Tavel JA, Oseekey K, McDermott CM, Mollerup D, et al. (2002) Regulatory approvals in a large multinational clinical trial: the ESPRIT experience. Control Clin Trials 23: 59-66.

8. Shah S, Whittle A, Wilfond B, Gensler G, Wendler D (2004) How do institutional review boards apply the federal risk and benefit standards for pediatric research? JAMA 4: 476-482.

9. Sherwood ML, Buchinsky FJ, Quigley MR, Donfack J, Choi SS, et al. (2006) Unique challenges of obtaining regulatory approval for a multicenter protocol to study the genetics of RRP and suggested remedies. Otolaryngol Head Neck Surg 135: 189-96.

10. Caulfield T, Ries N, Barr G (2011) Variation in ethics review of multi-site research initiatives. Ams Law Forum 2: 85-100.

11. Hadskis M (2011) The regulation of human biomedical research in Canada. In: Canadian Health Law and Policy. (4th edn), EdsDownie J, Caulfield, T, Flood C, LexisNexis Canada Inc, Ontario.

12. White MT, Gamm J (2002) Informed consent for research on stored blood and tissue samples: a survey of institutional review board practices. Account Res 9: 1-16.
13. Silverman $\mathrm{H}$, Hull SC, Sugarman J (2001) Variability among institutional review boards' decisions within the context of a multicenter trial. Crit Care Med 29 235-241.

14. Lenk C, Radenbach K, Dahl M, Wiesemann C (2004) Non-therapeutic research with minors: how do chairpersons of German research ethics committees decide? J Med Ethics 30: 85-87.

15. Ezzat H, Ross S, von Dadelszen P, Morris T, Liston R, et al. (2010) Ethics review as a component of institutional approval for a multicentre continuous quality improvement project: the investigator's perspective. BMC Health Serv Res 10: 223

16. Dyrbye LN, Thomas MR, Mechaber AJ, Eacker A, Harper W, et al. (2007) Medical education research and IRB review: an analysis and comparison of the IRB review process at six institutions. Acad Med 82: 654-660.

17. Palmour N, Affleck W, Bell E, Deslauriers C, Pike B, et al. (2011) Informed consent for MRI and fMRI research: analysis of a sample of Canadian consent documents. BMC Med Ethics 12: 1.

18. Racine E, Bell E, Deslauriers C (2010) Canadian research ethics boards and multisite research: experiences from two minimal-risk studies. IRB 32: 12-18.

19. Deslauriers C, Bell E, Palmour N, Pike B, Doyon J, et al. (2010) Perspectives of Canadian researchers on ethics review of neuroimaging research. J Empir Res Hum Res Ethics 5: 49-66.

20. Canadian Institutes of Health Research, Natural Sciences and Engineering Research Council of Canada,and Social Sciences and Humanities Research Council of Canada, Tri-Council Policy Statement: Ethical Conduct for Research Involving Humans, December 2010.

21. Enzel ME, Schmaltz R (2005) Ethics review of multi-centre clinical trials in Canada. Health Law Rev 13: 51-57. 